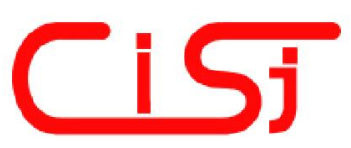

\title{
OBSERVATIONS-BASED COMPUTATIONAL ANALYTICS ON LOCAL CLIMATE DYNAMICS: CHANGE-POINTS
}

\author{
Yury Kolokolov, Anna Monovskaya \\ Orel State University, Komsomolskaya str. 95, 302026, Orel, Russia, www.oreluniver.ru \\ 2kolo@mail.ru, anna.monovskaya@mail.ru
}

\begin{abstract}
The paper concerns the problem on the computational decision making on evolution of local climate dynamics taking into account inevitable nonlinear nature of such systems and deficiency of reliable data on climate dynamics. With this purpose we consider annual temperature variation in the context of the bifurcation phenomena under the hysteresis regulation with double synchronization. The corresponding conceptual model (HDS-model) provides the homogeniety of the analysed states as well as the ability to derive some constituent of regional (external) impacts on a local climate system. In accordance with the peculiarities of HDS-model dynamics, we formalize three mechanisms of local temperature changes and introduce the corresponding indicators of change-points. It allows to substitute, at least partly, expert analytics concerning identification of qualitative changes in local climate dynamics. Examples of applications of the indicators are presented on the basis of processing the time series of temperature observations on daily mean surface air temperature made over last century. We believe that the results could be applied in order to increase the confidence of estimations about local climate changes. Copyright $(\mathbb{C}$ Research Institute for Intelligent Computer Systems, 2016. All rights reserved.
\end{abstract}

Keywords: Nonlinear time series, bifurcation analysis, nonlinear evolution, HDS-regulator, temperature observations, annual temperature variation, annual warming-cooling cycle, change point, tipping point.

\section{INTRODUCTION}

The paper is devoted to the discussion in the field of computational analytics on dynamics of local climate systems, where decision making is based on the processing instrumental observations taking into account inevitable nonlinear nature of such systems. In this case there is not exact conventional solution on how to provide the homogeneity of the analyzed states and processes $[1,2,3,4]$, where the essence of the problem consists in the following $[5,6]$ : how to identify qualitative changes in local climate dynamics? And there are at least three widespread notions which are associated with "qualitative changes", namely: abnormalities; change points; bifurcation points. In particular, various weatherclimate abnormalities represent deviations from ordinary behaviors or from changes in these behaviors expected in accordance with the traditional statistical conceptions [7, 8]. For example, an "abnormality" can mean a heightened deviation from an average value, a change in direction and/or rate of a trend, some strange event, and so on. In other words, there are a lot of facts which demonstrate insufficiency of only statistical description of climate dynamics in order to provide the homogeniety of the analysed states $[1,2,6,8]$.

One of the most promising ways towards advanced descriptions of climate dynamics is connected with attracting conceptions of the bifurcation analysis $[5,6,9]$, which remains the unique tool to identify reasonably qualitative changes connected with a change of a type of a dynamical process: from one periodical process to another one; from a periodical process to a chaotic one; and so on. However, the main trouble to introduce computational analytics based on the bifurcation analysis into practical applications remains connected with too great part of expert intelligence while decision-making [10, 11, 12]. Especially, it occurs in the case of complex nonlinear phenomena, for example, intermittency phenomena [13]. Additionally, it is necessary to take into account that incomplete and inexact data on climate variables form one of the most actual challenges in the present-day climatology [7, 9]. That is why non-adapted bifurcation analysis seems to be quite hardly applied $[6,9]$.

So, the third notion connected with qualitative changes in local climate dynamics seems to be the 
most appropriate in this context. Initially, the notion of a change-point was rather empirical and was based on the following considerations [5, 14]: a climate system represents a nonlinear object; so, it is supposed that appearance of a strangeness should be somehow connected with a significant change in dynamics (conceivably, it could be connected with a bifurcation). In particular, any idea concerning a change-point for nonlinear system dynamics has originally an expert constituent, which allows to adapt the known method for a novel ability in application to a specific task only $[13,15,16]$. In other words, it seems to a a non-trivial question on how conceptions of the bifurcation analysis could be transformed by expert intelligence into some adapted indicators of nonlinear evolution in order to realize computationally decision-making on a certain point at least. In particular, here we focus on both local and regional reasons of the resultant local temperature changes, where bifurcation phenomena form one of the mechanisms.

The conceptual model of a hysteresis regulator with double synchronization (HDS-regulator) is used to describe peculiarities of annual temperature variation in order to provide different aspects of homogeniety of the analysed states $[6,9,17,18]$. Brief comments concerning peculiarities of this model in compartison with the existing ones are presented in section 2. Then, three specialized indicators on change-points are introduced together with the mechanizms of local temperature changes (section 3). Then, we discuss the novel method on how to apply these indicators in order to exhibit interrelation over time between local and regional effects leading to temperature changes (section 4). Main results and future outlook are presented in section 5. The results are based on processing the open-access data of the meteorological observation on daily mean surface air temperature over last 100130 years provided by Russian Research Institute of Hydrometeorological Information - World Data Center (www.meteo.ru).

\section{UNI- AND MULTI-BEHAVIOR MODELS OF LOCAL TEMPERATURE DYNAMICS}

At present, there are three conceptually different viewpoints on description of annual temperature variation. Let us explain briefly the essence of this difference. In this connection it is necessary to pay attention on the following fact $[1,3,4,18,19]$ : variability of both duration and beginning date observed in dynamics of annual temperature cycles in relation to an year from $1^{\text {st }}$ January to the next one. For example, several typical cases are illustrated in Fig. 1a. The first viewpoint represents an "external" description of the realized annual cycles that is grounded on the following hypothesis: the observed variability can be described by one averaged annual behavior with deviations from mean values caused by chaotic nature. Units of such averaged behavior are made by regular fragmentation of the temperature time series from one $1^{\text {st }}$ January to the next one (dotted vertical lines in Fig. 1a), and deviations are statistically determined. In other words, it is a uni-behavior model of a local climate system, where such behavior is denoted by the notion of "annual temperature variation" (hereafter ATV-unit, Fig. 1a,b). The model remains the basic one in order to determine climate norms, to analyze climate changes and to assess climate risks [7, 20, 21, 22].

However, there are too much abnormalities in relation to which it was shown that such direct statistical description can be incorrect and incomplete $[1,2,17,18]$. In this connection, the second viewpoint develops the "external" description in order to try to cover the observed variability and/or to determine some cause-effect relations by means of advanced statistical processing $[3,4]$, by means of specialized embedded structures [19], and so on. Nevertheless, regularities, on why and how the observed abnormal temperature variability is formed, were not revealed from the "external" description till now. We believe that the main problem is connected with heterogeneity of the analyzed annual cycles, to which the statistical hypothesis can not be originally applied $[6,9]$. Then the following question appears: how to reason clustering these cycles into homogeneous groups? In this connection we proposed to attract the bifurcation analysis [17]. As a result, the third viewpoint appears to discuss $[6,9]$, where "internal" description, on why and how the abnormal variability occurs, is developed from the inherent peculiarities of HDS-regulator dynamics.

The corresponding hypothesis on local climate dynamics (HDS-hypothesis) assumes that each year a local climate system can follow one of three elemental behaviors; and alternations between these behaviors form a unique pattern of this local system dynamics evolution over time. In other words, it is a multi-behavior model of a local climate system. The elemental behaviors were denoted by $R-, L-$ and $C$ behaviors due to right, left and central locations of the temperature maximums correspondingly (Fig. 1c). These geometrical peculiarities are naturally formed in accordance with the control algorithm on the basis of the competition between the level and time quantizations $[6,17,18]$. As a result, units of the multi-behavior model are made by irregular fragmentation of temperature time series from one minimum to the next one (Fig. 1a,b). Such time series fragment includes full warming stage and 
full cooling stage that was denoted in $[17,18]$ as annual warming-cooling cycle (AWCC-unit).
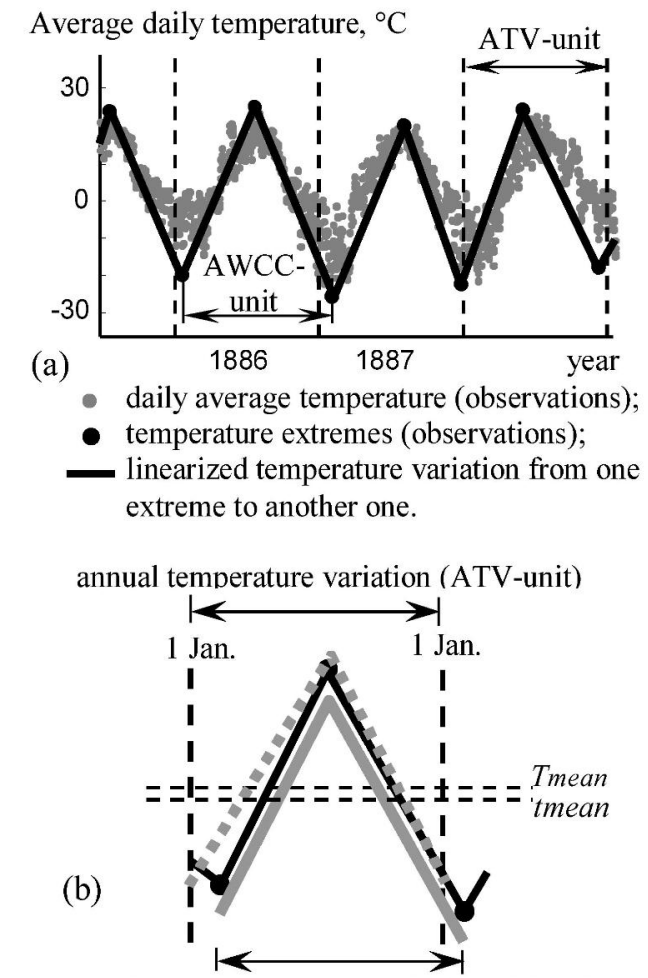

annual warming-cooling cycle (AWCC-unit)

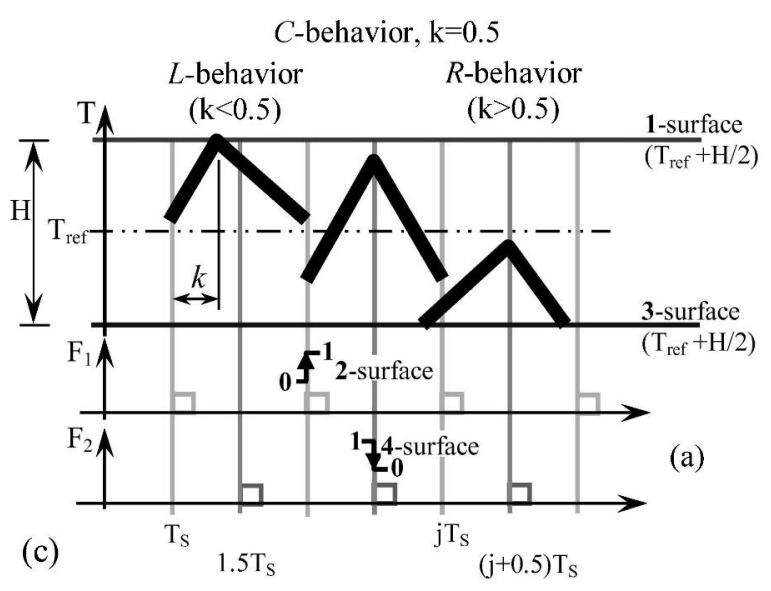

$H$ is the local temperature hysteresis;

$T_{\text {ref }}$ is the local temperature reference;

$T_{S}$ is the synchronization period ( 1 year); $\mathrm{j}=0,1,2, \ldots$; $k$ is the relative duration of warming stage to $T_{S}$-period; $L$-behavior is formed by 2-1-4-2-sequence of the sewing surfaces; $C$-behavior is formed by 2-4-2-sequence of the sewing surfaces; $R$-behavior is formed by 3-2-4-3sequence of the sewing surfaces.

Fig. 1 - A part of temperature observations to show regular and irregular interannual fragmentations (a); treatments of ATV-unit and AWCC-unit (b); scheme to illustrate HDS-regulator dynamics (c)

Alternations between $R$-, $L$ - and $C$-behaviors (i.e. intermittency) are caused by border collision bifurcations, where, mostly, the following long lasting combinations were identified in dynamics of real climate systems $[6,17]: L C$-intermittency, $R C$ intermittency, and $R L C$-intermittency.

So, on the one hand, $C$-behavior seems to be look like annual temperature variation; and $C$-behavior is dominant in local climate dynamics. It explains why the uni-behavior model remains till now the most widespread and convenient for the habitual thinking. On the other hand, the multi-behavior model allows to explain in coordination many observed abnormalities $[9,17]$ as opposed to the uni-behavior model; however, it supposes novel notions and processes which were not earlier considered. In particular, in the case of ATV-units, time coordinates are fixed and adjusted, and any built-in regulator is absent. Mainly, average annual temperature $\left(T_{\text {mean }}\right.$, Fig. $\left.1 \mathrm{~b}\right)$ is analyzed; less often, temperature extremes are taken into account with monthly resolution. In the case of AWCC-units, time coordinates are unfixed and should be identified; and the built-in regulator accompanies the model. And essentially more characteristics are always analyzed with daily resolution, namely (Fig. 1b): average temperature (tmean); time and temperature coordinates of the both extremes; amplitude and relative duration of warming stage ( $k$, Fig. 1c), and so on. Moreover, novel notions connected with the built-in regulator are additionally taken into account: a local temperature reference (Tref) and a local temperature hysteresis $(H$, Fig. 1c).

\section{INDICATORS FOR TEMPERATURE CHANGES}

Strictly speaking, none of ATV-unit characteristics corresponds to none of AWCC-unit characteristics: $T_{\text {mean }^{-}}$and tmean-values are approximately the same; the rest characteristics either can be essentially different due to different scales used to calculate its values or can not be even compared in sense. That is why any idea associated with processing AWCC-units supposes the necessity to formalize expert analytics on a novel subject by the corresponding method, and to assess whether there are advantages of this method in comparison with the traditional processing ATV-units. In particular, let us focus on the AWCC-unit characteristics which describe reasons leading to changes of the average annual temperature.

First, any local climate system can choice one of three kinds of behaviors each year (Fig. 2a), where $L$-behavior is comparatively "warm", $R$-behavior is comparatively "cool", and $C$-behavior is comparatively neutral (warmer than $R$-behavior and colder than $L$-behavior). The notion of "comparatively" means that variation of the average 
temperature (tmean-variation, Fig. 2a) can be realized in the context of the fixed local $H$-limit and Tref-level. So, the first reason, in accordance to which changes of average annual temperature could be naturally realized, is connected with bifurcation phenomena (namely, intermittency). Such changes relate only to local climate peculiarities, i.e. can be realized without regional or global climate changes. Since $k$ (Fig. 1c) is the main bifurcation parameter $[6,17]$, then evolution of $k$-values shows in what extent the state of a certain local climate system is stabilized. So, let $d k$ is a difference between the running and previous $k$-values. Then the smaller $d k$ values are, the smaller changes of average annual temperature are. For example, $d k$-evolution determined by processing the surface air temperature observations made in St-Petersburg is presented in Fig. 2b.
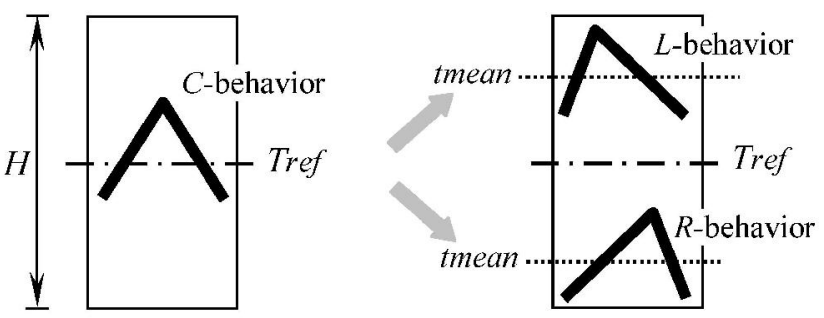

(a)

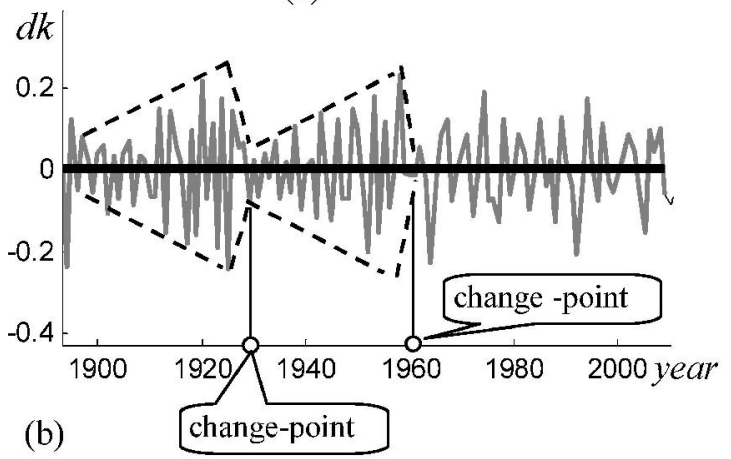

- $d k$-evolution determined by processing the temperature observations;

- zero-level to indicate a stabilized state.

Fig. 2 - Scheme to explain a mechanism of local temperature evolution described by $d k$-indicator (a); results of $\boldsymbol{d} k$-analysis made by processing the temperature observations.

Second, in accordance with the HDS-hypothesis, temperature changes can be naturally realized with changes of the temperature hysteresis $(H)$ only, when the rest circumstances are not changed. In this case, physical essence of $H$-evolution is associated with a component of relative regional solar radiation [23]. For example, let a local climate system is characterized by the warmest state within its own local context (i.e. it is $L$-behavior about up temperature level, Fig. 3a). However, the bigger change in $H$-value becomes, the bigger change in the average annual temperature becomes without bifurcation phenomena. In particular, decrease of the running tmean-value can be realized with the potentially warmest states in the context of own resources of the same local climate system (Fig. 3a, upper part, before and after $H$-decrease). And vice versa, increase of the running tmean-value can be realized with the potentially coldest states (Fig. 3a, low part, before and after $H$-decrease). In particular, $H$-evolution determined for St-Petersburg is presented in Fig. $3 b$.

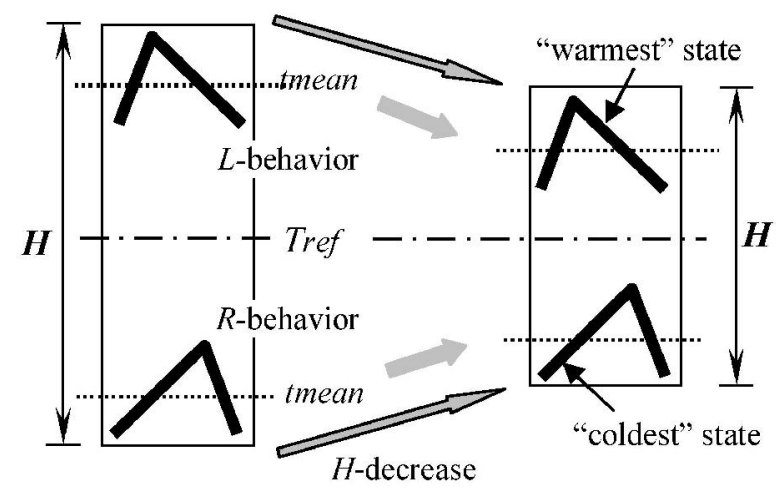

(a)

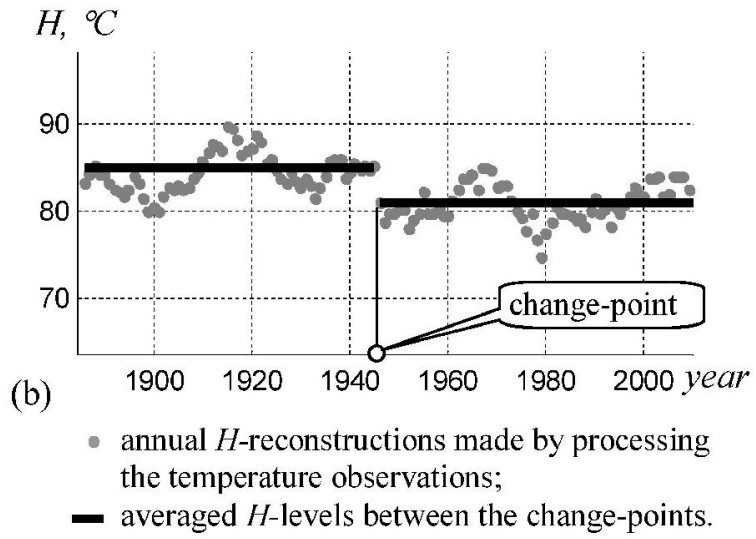

Fig. 3 - Scheme to explain a mechanism of local temperature evolution described by $\boldsymbol{H}$-indicator (a); results of $\boldsymbol{H}$-analysis made by processing the temperature observations.

Third, in accordance with the HDS-hypothesis, temperature changes can be naturally realized with changes of the temperature reference (Tref) only, when the rest circumstances are not changed. In this case Tref is associated with a component of relative regional temperature peculiarities between comparatively warm and comparatively cold quasihomogeneous climate regions [22, 23]. For example, let a local climate system is characterized by the coldest state within its own local context (i.e. it is $R$ behavior about the low temperature level, Fig. 4a). 
However, the higher Tref-value is, the bigger increase in the average annual temperature becomes in spite of the potentially coldest states from the own resources of this local climate system. And vice versa, the lower Tref-value is, the bigger decrease in the average annual temperature becomes in spite of the potentially warmest states from the own resources of this local climate system. In particular, Tref-evolution determined for St-Petersburg is presented in Fig. $4 b$.
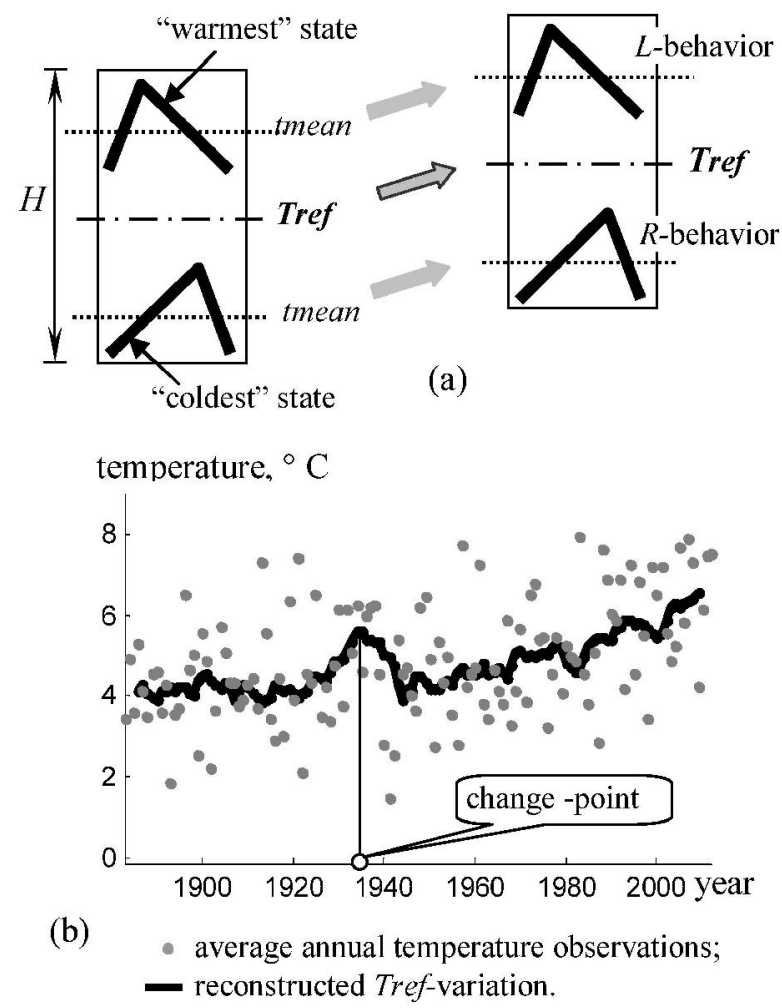

Fig. 4 - Scheme to explain a mechanism of local temperature evolution described by Tref-indicator (a); results of Tref-analysis made by processing the temperature observations.

\section{METHOD OF CHANGE-POINTS}

Increase in annual average temperature (first of all, in global scale) remains the leading characteristic to show the result of climate changes $[7,20,21,22]$. With this purpose local changes are generalized to the regional one, and, after, regional changes are generalized to the global one. The main feature of the discussed viewpoint consists in the following: in accordance with the dynamics of the HDS-regulator it is possible to separate quite definitely local and regional reasons which lead to the resultant temperature changes. With this purpose each of these reasons is described by the corresponding indicator (Figs. 2a, 3a, 4a), evolution of which can be visualized (Figs. 2b, 3b, 4b) in order to identify change points at least.

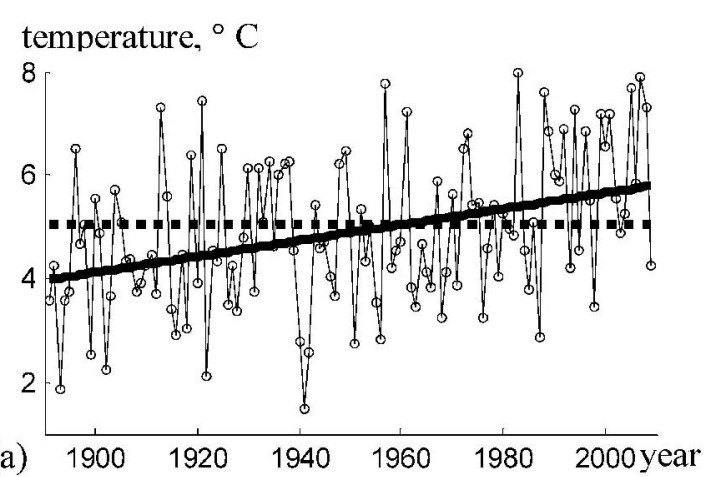

- average annual temperature (observations)

- with visualization of time series profile;

-." climate norm determined over 1961-1990 years;

simple linear trend.

temperature, ${ }^{\circ} \mathrm{C}$

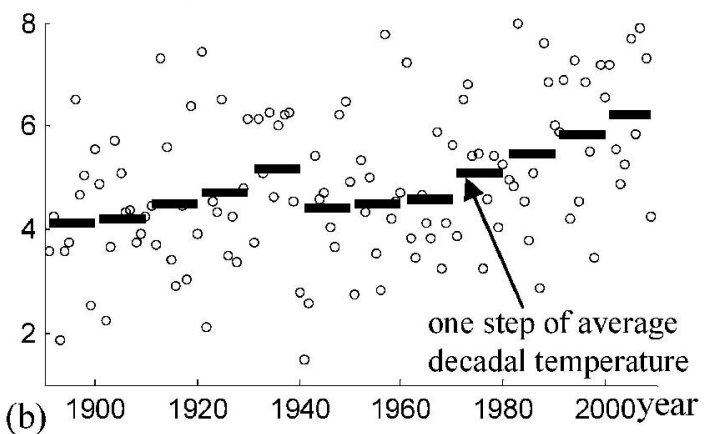

temperature, ${ }^{\circ} \mathrm{C}$

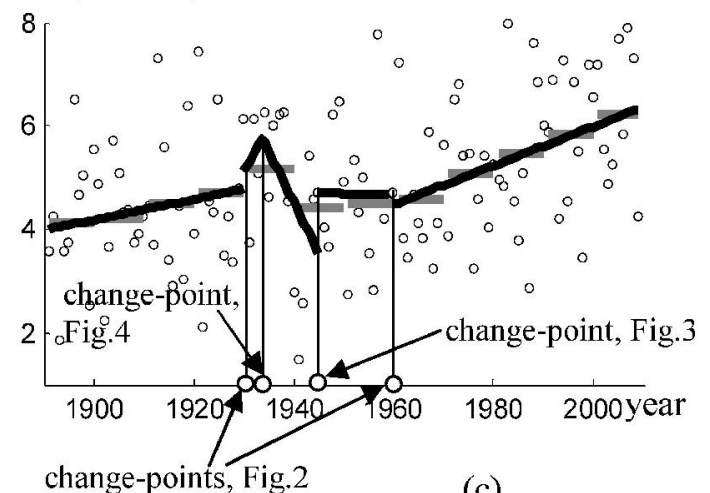

change-points, Fig.2 (c)

$\circ$ change-point;

- piece-wise linear trend between neighboring change-points;

- one step of decadal temperature averaging.

Fig. 5 - Simple trend estimating in relation to the climate norm of 1961-1990 years (a); average decadal smoothing (b); piece-wise trends between the change points (c) combined with the average decadal smoothing.

So, the idea of the proposed method consists in the following: each indicator translates one or several change points in dynamics of local climate system separately; next, all translated change points are jointed; then, the carrier states through which a local climate system passed can be visualized by trends between these change-points. In other words, the method is intended for formalized identification 
of change points in order to analyze dominant trends in local and regional temperature dynamics.

Let us consider two examples of such analytics. Let us compare trend analysis of average annual temperature (denoted by small circles) made by traditional (Fig. 5a,b) and the proposed (Fig. 5c) ways. In the first case, two variants are used [7, 22]: linear trend over the whole considered period (Fig. 5a, bold black line) in relation to the climate norm over 1961-1990 years (Fig. 5a, dotted black line); decadal averaging synchronized with beginnings and ends of centenary decades (Fig. 5b, bold horizontal black lines). In the second case we joint the change-points determined for three indicators. For example, this combination can be presented against the background of Tref-evolution (Fig. 5c). Then, we build linear trends between the identified change-points (Fig. 5c, bold horizontal black lines). These trends are quite cloze to decadal smoothing beyond the range of change-points (denoted by gray horizontal black lines in Fig. 5c), and, additionally, these trends allow to exhibit breaks, each of which is caused by the change of one or several mechanisms of local temperature evolution (Figs. 2a, 2b, 2c). Let us comment it.

Till now, we consider visualization of temperature changes in own scales of each of the analyzed indicator. Let us combine $H$-, Trefevolution (Fig. 6a), then resultant dynamics needs in zoom-in (zoom-in fragments in Fig. 6a) in order to be visualized similar to the schemes (Figs. 2b, 3b, 4b). Namely, there are two transients, where the first one is completed (Fig. 6a, transient from state 1 to state 2); the second one continues (Fig. 6a, transient from state 2 to state 3). During the first transient $H$ - decrease dominates (in accordance with the mechanism described in Fig. 3a), as a result of which Tref-value returns to practically the same one, and the local bifurcation activity achieves its maximal $L C$-level (Fig. 6b). During the second transient Tref-increase dominates (in accordance with the mechanism described in Fig. 4a), as a result of which $H$-value is stabilized, and the local bifurcation activity decreases monotonously (Fig. 6c). As a rule, several mechanisms effect jointly on local climate dynamics; sometimes, one of the mechanisms can be accepted as dominant one (as it is illustrated in Fig. 6b,c); nevertheless, changepoints can be identified independently for each of the indicators.

So, the presented method of change-points provides the possibility to divide reasonably a time series of temperature observations with a complex profile (for example, time series of the average annual temperature in Fig. 5a) into sections, within which evolutional transients can be encapsulated. Such division is reasoned by peculiarities of HDS- dynamics similar to the specialized bifurcation analysis [6, 9, 17]; but, unlike the bifurcation analysis, such division can be fully formalized (without stages of expert decision-making).
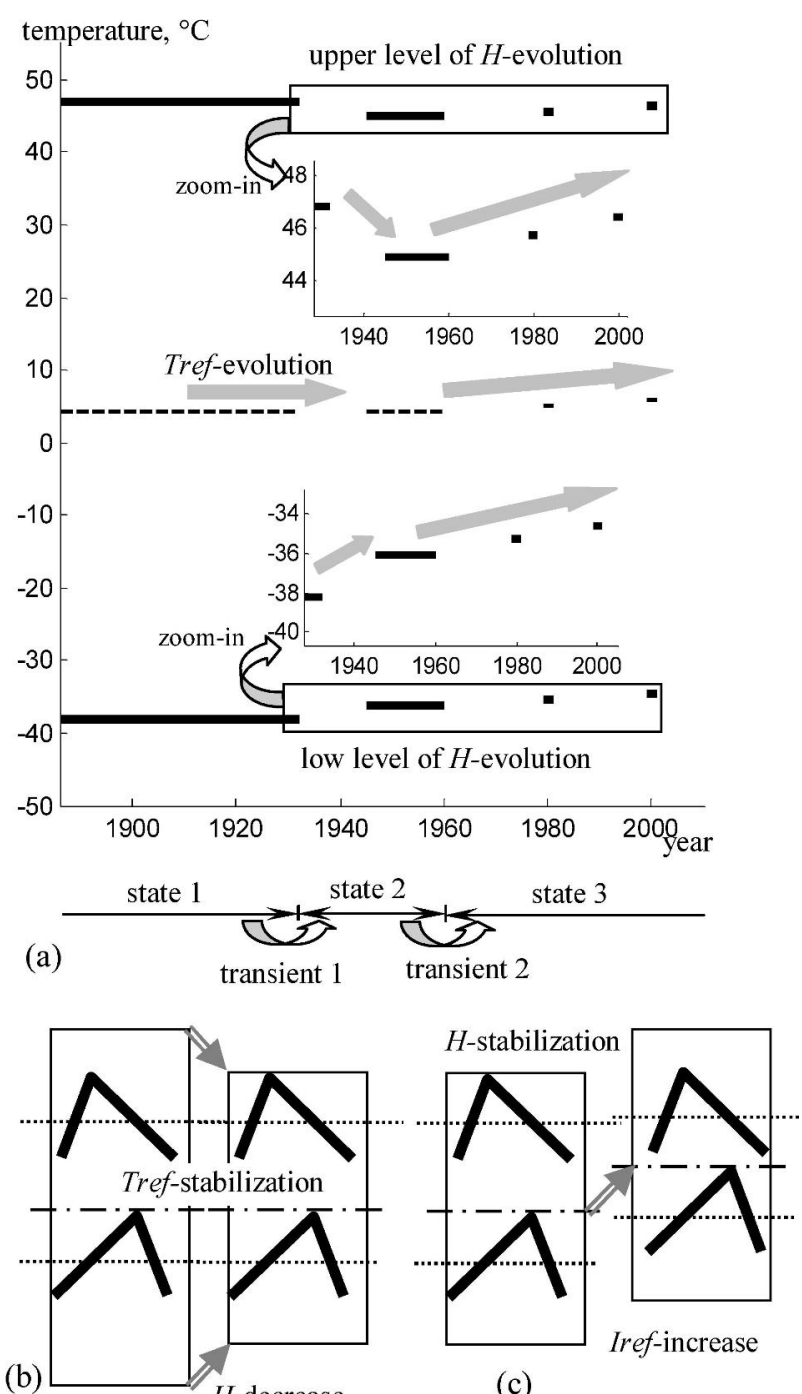

Fig. 6 -Reconstructed combination of $\mathrm{H}$-, Trefevolution (a); comments to transients with dominant $H$-decrease (b) and dominant Tref-increase (c).

\section{CONCLUSION}

The discussed method of analysis of time series of local temperature observations belongs to the groups of methods grounded on the conceptual model of local climate dynamics, where the observed abnormalities of annual temperature variability are excused by intermittency between three kinds of annual behavior $[6,9,17,18]$. Such methods are focused on the bifurcation analysis and detailed reconstruction of local climate dynamics; here we focus on both local and regional reasons of the resultant local temperature changes, where bifurcation phenomena form only one of the mechanisms. In particular, indicators of three mechanisms are established, in accordance to which 
the change-points can be identified in the context of time series with a complex profile. These changepoints seem to be useful in application to different trend estimations, and such application is restricted by the physical sense of the proposed indicators in the context of the HDS-hypothesis on local climate dynamics. At the same time, there is a peculiarity of the method which could be interesting in future research, namely: an ability to derive some constituent of regional (external) impacts on a local climate system by processing its (local) temperature time series opens a promising way to avoid the deficiency of data on regional dynamics. In other words, it opens a way how to get additional useful information by processing the data which are incomplete and irreparable.

\section{REFERENCES}

[1] S. Pezzulli, D. B. Stephenson and A. Hannachi, "The variability of seasonality," Journal of Climate, Vol. 18, pp. 71-88, 2005.

[2] Ch. Essex, "Climate theory versus a theory for climate," International Journal of Bifurcation and Chaos, Vol. 21, pp. 3477-3487, 2011.

[3] M. Hoerling, A. Kumar, R. Dole, J. W. Nielsen-Gammon, J. Eischeid, J. Perlwitz, X.-W. Quan, T. Zhang, P. Pegion and M. Chen, "Anatomy of an extreme event," Journal of Climate, Vol. 26, pp. 2811-283, 2013.

[4] P. Huybers, K. A. McKinnon, A. Rhines and M. Tingley, "US daily temperatures: The meaning of extremes in the context of nonnormality," Journal of Climate, Vol. 27, pp. 7368-7384, 2014.

[5] J. M. Thompson and J. Sieber, "Predicting climate tipping as a noisy bifurcation: A review," International Journal of Bifurcation and Chaos, Vol. 21, pp. 399-423, 2011.

[6] Yu. Kolokolov and A. Monovskaya, "Guesswork and reasonings on centennial evolution of surface air temperature in Russia. Part II: Is it possible to research both local peculiarities and regional tendencies from the bifurcation analysis viewpoint?," International Journal of Bifurcation and Chaos, Vol. 26, 1650071, 2016.

[7] T.F. Stocker, et al. (eds.) "IPCC 2013: Summary for policymakers," Climate Change 2013: The Physical Science Basis. Contribution of Working Group I to the Fifth Assessment Report of the Intergovernmental Panel on Climate Change (Cambridge University Press, Cambridge), Chapter SPM, pp. 3-29, 2013.

[8] B. Christiansen, "Changes in temperature records and extremes: Are they statistically significant?," Journal of Climate, Vol. 26, pp. 7863-7875, 2013.

[9] Yu. Kolokolov and A. Monovskaya, "Guesswork and reasonings on centennial evolution of surface air temperature in Russia. Part IV: Towards economic estimations of climaterelated damages from the bifurcation analysis viewpoint?" International Journal of Bifurcation and Chaos, Vol. 26, 1630033, 2016.

[10] C.P. Silva, Nonlinear dynamics and chaos: from concept to application, 2011, [Online]. Available: http://www.aerospace.org/cross linkmag/spring-2011/nonlinear-dynamics-andchaos-from-concept-to-application/).

[11] H. S. Karagueuzian, H. Stepanyan and W. J. Mandel, "Bifurcation theory and cardiac arrhythmias," American Journal of Cardiovascular Disease, Vol. 3, pp.1-16, 2013.

[12] Yu. Kolokolov, S. Koschinsky and A. Hamzaoui, "Comparative study of the dynamics and overall performance of boost converter with conventional and fuzzy control in application to PFC," in Proceedings of the $9^{\text {th }}$ IEEE Power Electronics Specicalist Conference, Aachen, Germany, June 20-26, 2004, pp. 2165-2171.

[13] Yu. Kolokolov and A. Monovskaya, "From modifications of experimental bifurcation diagrams to operating process stability margin," International Journal of Bifurcation and Chaos, Vol. 23, 1330024, 2013.

[14] J. A. Rial, Sr. R. A. Pielke, M. Beniston, M. Claussen, J. Canadell, P. Cox, H. Held, N. de Noblet-Ducoudre, R. Prinn, J. F. Reynolds and J. D. Salas, "Nonlinearities, feedbacks and critical thresholds within the Earth's climate system," Climatic Change, Vol. 65, pp. 11-38, 2004.

[15] C. M. Duarte, S. Agusti, P. Wassmann, J. M. Arrieta, M. Alcaraz, A. Coello, N. Marba, I. E. Hendriks, J. Holding, I. Garcia-Zarandona, E. Kritzberg and D. Vaque, "Tipping elements in the arctic marine ecosystem, $A M B I O$, Vol. 41, pp. 44-55, 2012.

[16] M. S. Williamson, S. Bathiany and T. M. Lenton, "Early warmung signals of tipping points in periodically forced systems," Earth System Dynamics, Vol. 7, pp. 313-326, 2016.

[17] Yu. Kolokolov and A. Monovskaya, "Modified bifurcation diagrams to analyse the intermittency observed in local climate dynamics," in Proceedings of the $8^{\text {th }}$ IEEE International Conference on Intelligent Data Acquisition and Advanced Computing Systems: 
Technology and Application, IDAACS'2015, Warsaw, Poland, September 24-26, 2015, pp. 624-630.

[18] Yu. Kolokolov and A. Monovskaya, "Multidimensional analysis of dynamics of annual warming-cooling cycles on the basis of index model of temperature observations," in Proceedings of the $8^{\text {th }}$ IEEE International Conference on Intelligent Data Acquisition and Advanced Computing Systems: Technology and Applications IDAACS'2015, Warsaw, Poland, September 24-26, 2015, pp. 631-637.

[19] J. Feng, Z. Wu, and G. Liu, "Fast multidimensional ensemble empirical mode decomposition using a data compression technique," Journal of Climate, Vol. 27, pp. 3492-3504, 2014.

[20] Documents of the 16th Session of the Commission for Climatology (CCl-16), Heidelberg, Germany, July 3-8, 2014: CCL16/Doc.8.1. Final Report with resolutions and recommendations (WMO-No.1137, World Meteorological Organization, Geneva, Switzerland).

[21] Valuing Weather and Climate: Economic Assessment of Meteorological and Hydrological Services, 2015 (WMO-No. 1153, World Meteorological Organization, Geneva, Switzerland).

[22] J. Blunden and D. S. Arndt, Eds., 2016: State of the Climate in 2015. Bulletin of American Meteorological Society, Vol. 97, Issue 8, S1S275, 2016.

[23] K. E. Trenberth and J. T. Fasullo, "Regional energy and water cycles: Transports from ocean to land," Journal of Climate, Vol. 26, pp. 7837-7851, 2013.

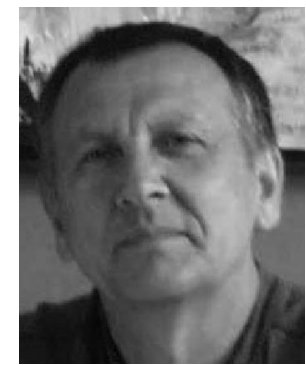

Yury Kolokolov received M.S. degree in 1972 (Tomsk Politechnical Institute, Russia), PhD degree in 1978 (Tomsk Politechnical Institute, Russia), Russian academic degree of Senior Doctor in 1990 (Tomsk University of Control and Radioelectronics, Russia). From 1993 to 2007 he was a full professor and Head of the Dept. of Design and technology of electronic systems (State Technical University of Orel, Russia); from 2007 to 2012 he was a full professor and Director of Institute of Control Systems and Information Technologies (UGRA State University, Khanty-Mansiysk, Russia); from 2012 to 2016 he was a chief researcher and a Head of Dept. of Applied Scientific Research (Russian Research Institute of Hydro meteorological Information - World Data Center, Obinsk, Russia). Currently he is a Head of scientific school (Orel State University, Russia). His scientific interests include theory, experiment and practice of nonlinear dynamics in the field of complex systems.

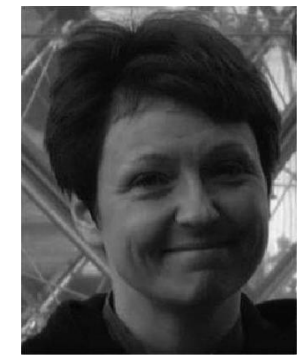

Anna Monovskaya received M.S. degree in 2001 (State Technical University of Orel, Russia), PhD degree in 2005 (State Technical University of Orel, Russia and University of Technology of Troyes, France), Russian academic degree of Senior Doctor in 2010.

Currently, she is a full professor and a Head of a Scientific-Educational Center (Orel State University, Russia). Her scientific interests include theory, experiment and practice of nonlinear dynamics in the field of complex systems. 